\title{
A Combined Denoising Method for Microseismic Signals from Coal Seam Hydraulic Fracturing: Multithreshold Wavelet Packet Transform and Improved Hilbert-Huang Transform
}

\author{
Zhengxing Yu, ${ }^{1}$ Jinglin Wen $\mathbb{C D}^{1}{ }^{1}$ Quanjie Zhu $\mathbb{D}^{\circ},{ }^{2}$ Haitao Ma, ${ }^{1}$ and Yu Feng ${ }^{3}$ \\ ${ }^{1}$ China Academy of Safety Science and Technology, Beijing 100012, China \\ ${ }^{2}$ School of Safety Engineering, North China Institute of Science and Technology, Beijing 101601, China \\ ${ }^{3}$ Department of Civil Engineering, University of Toronto, Ontario M5S 1A4, Canada \\ Correspondence should be addressed to Jinglin Wen; wenjl@chinasafety.ac.cn
}

Received 26 October 2020; Revised 26 December 2020; Accepted 31 January 2021; Published 20 February 2021

Academic Editor: Junfei Zhang

Copyright $(2021$ Zhengxing Yu et al. This is an open access article distributed under the Creative Commons Attribution License, which permits unrestricted use, distribution, and reproduction in any medium, provided the original work is properly cited.

Coal seam hydraulic fracturing (CSHF) has recently been applied to mitigate frequent regional rockburst risk in deep mines before mining practice, as an effective substitute for conventional labor-intensive and time-consuming rockburst prevention measures. Due to the complex nature of CSHF microseismic signals-e.g., nonstationary, transient, and low signal-to-noise ratio-conventional denoising methods tend to yield undesirable results that may preclude reliable evaluation of hydraulic fracturing performance using microseismic data. We propose an advanced denoising method MWPT-IHHT to achieve twice denoising in a fine and adaptive manner. This method combines a multithreshold wavelet packet transform (MWPT) and an improved Hilbert-Huang transform (IHHT), with each being improved compared to their conventional counterparts. A quantitative comparison using synthetic signals suggests the outperformance of the proposed method over the commonly used denoising methods in suppressing noises in terms of signal-to-noise ratio, signal similarity, and energy percentage. The desirable denoising results of two typical real CSHF signals in a CSHF test at Huafeng Coal Mine further demonstrate the applicability and effectiveness of the proposed MWPT-IHHT method.

\section{Introduction}

With coal resources being continuously extracted for decades, a great number of coal mines in China have entered into deep mining, and as a result, rockbursts have become one of the most dangerous dynamic hazards, causing tremendous economic losses and casualties [1-5]. To date, around 180 rockburst mines have been identified, with over 50 of them currently mining at a critical depth of approximately $1000 \mathrm{~m}$ [4-6]. Even worse, some of these mines are being subjected to complex geological and mining conditions, which cause more frequent and intense rockbursts, e.g., faults, island longwall mining, and inappropriate mine design. Conventional rockburst prevention measures that involve a small volume, e.g., destress drilling and destress blasting, have long been applied and can effectively control local rockbursts in highly stressed zones. However, these measures are both labor- intensive and time-consuming, which renders them less suitable for deep mines subjected to frequent rockburst hazards. Therefore, a cost-effective technique for regional rockburst prevention is urgently needed.

To this end, hydraulic fracturing, initially used for oil and gas stimulation and in situ stress measurement [7-9], has recently been applied to mitigate frequent rockburst risk in deep mines through preconditioning hard roofs and coal seams before mining practice $[6,10-12]$. However, further development of this technique has been restricted due to the absence of a mine-specific method for evaluating the hydraulic fracturing performance using microseismic monitoring data, and consequently, guidelines for determining appropriate design parameters for hydraulic fracturing treatment are underdeveloped.

Signal denoising is particularly crucial in signal processing as extraction of clean and valid signals is a prerequisite for 
reliable evaluation of hydraulic fracturing performance. Valid signals that are smeared in the ambient noises are often neglected if no appropriate denoising methods are available. Conventional denoising methods include short-time Fourier transform, Wigner-Ville distribution, S-transform, wavelet transform, wavelet packet transform (WPT), and HilbertHuang transform (HHT) [13-17]. These time-frequency methods have demonstrated their desirable capabilities of reducing noises in nonstationary signals, and currently WPT, HHT, and their respective variations are most widely used for processing signals in geotechnical, mining, oil and gas, and seismic exploration fields. Zhu et al. [18] investigated the energy distribution characteristics for two common types of microseismic signals (i.e., blasting-induced and roof-breakinduced signals) in mines using WPT and proposed a quantitative method for distinguishing the two signals. The authors in [19] employed artificial neural network and WPT to suppress random noise in seismic signals. The authors in [20] applied HHT to seismic reflection data to identify the instantaneous attributes in order to develop a superior filter for enhancing the signal-to-noise ratio. The authors in [21] examined the rationale of HHT for analyzing dynamic and earthquake motion recordings in studies of seismology and engineering and demonstrated its capability of extracting the time-frequency energy distribution characteristics, which might not be exposed effectively and efficiently by Fourier transform technique. However, WPT-based and HHT-based methods are not wellsuited for more complex signals, even rendering signal distortion and damage in some cases, i.e., transient, nonstationary, and low energy microseismic signals with low signal-to-noise ratio from coal seam hydraulic fracturing (CSHF). Therefore, an advanced denoising method for this particular type of complex signal is needed in order to properly evaluate CSHF performance.

We previously developed a preliminary framework for processing and interpreting microseismic monitoring data obtained from a CSHF test at Huafeng Coal Mine in China, including improved HHT signal denoising, automatic first arrival picking, and four-channel combined source locating [6]. Following this work, this paper aims to extend and further develop the denoising method for CSHF signals by combining a multithreshold wavelet packet transform (MWPT) and an improved Hilbert-Huang transform (IHHT), with each being improved compared to the conventional methods and then combined for twice denoising. A comparison using numerically synthetic data is made between the proposed method MWPT-IHHT and the three other commonly used denoising methods, and it shows the outperformance of the MWPT-IHHT in reducing noises in terms of signal-to-noise ratio, signal similarity, and energy percentage. The application using real CSHF signals further demonstrates the applicability and effectiveness of the proposed denoising method for processing CSHF signals.

\section{Characteristics of Microseismic Signals in Coal Seam Hydraulic Fracturing}

Figure 1 shows two typical microseismic signals with low and high signal-to-noise ratio SNR observed in the CSHF test at Huafeng Coal Mine, and more information on this test can be found in [6]. In the time domain, the two valid signals have peak amplitudes of $13.6 \mathrm{mV}$ and $131.8 \mathrm{mV}$ with a duration of only $150 \mathrm{~ms}$ and $200 \mathrm{~ms}$, respectively. In order to better understand the characteristics of CSHF signals, the two signals are further displayed in the frequency domain through Fourier transform technique. The frequency domain is displayed into two parts corresponding to the front waveform mainly containing noises and the rear waveform containing the valid signal. For the signal with low SNR in Figure 1(a), the frequency of the valid signal mainly concentrates at roughly $75 \mathrm{~Hz}$, while two regular frequency concentrations are observed in the background noises at approximately $170 \mathrm{~Hz}$ and $335 \mathrm{~Hz}$, respectively. The regular concentrations might be caused by the pulse of high-pressure water in the tube. For the signal with high SNR in Figure 1(b), the valid signal mainly concentrates in the frequency band of 50-200 Hz. Combining the same analysis for many other observed signals, the dominant frequency of valid CSHF signals in this test is mainly distributed between 30 and $200 \mathrm{~Hz}$.

For the signal with low SNR, the peak amplitude is $13.6 \mathrm{mV}$, while the background noises have comparable magnitudes of approximately $3 \mathrm{mV}$. In addition, its frequency magnitude is even lower than that of background noises. In this case, the valid signal appears to be smeared in the background noises, and it is also observed that most CSHF signals possess a low signal-to-noise ratio. This renders accurate first arrival picking, source locating, and further performance evaluation rather difficult and challenging.

Through the above analysis, it is found that the CSHF signals are transient, nonstationary, low in energy, and low in SNR with a dominant frequency band of $30-200 \mathrm{~Hz}$. For this type of signals, the commonly used denoising methods-WPT and HHT-can cause signal distortion arising from the lack of adaptivity and insufficient resolution in the frequency domain, and this may yield undesirable denoising results. Therefore, to overcome the shortcomings of the conventional methods in processing CSHF signals, a method named MWPT-IHHT is proposed to achieve a twice denoising, which combines a multithreshold WPT and an improved HHT.

\section{Combined MWPT-IHHT Denoising Method}

MWPT-IHHT denoising method employs a multithreshold wavelet packet transform to implement preliminary denoising and an improved Hilbert-Huang transform for a second denoising. The combination of MWPT and IHHT, with each being improved compared to their conventional counterparts, can significantly enhance the time-frequency localization and allow complex signals (CSHF signal) to be processed in a fine and adaptive manner.

3.1. Multithreshold Wavelet Packet Transform. Wavelet packet transform (WPT) was proposed by $[22,23]$ as a generalization and improvement of wavelet transform, with the 


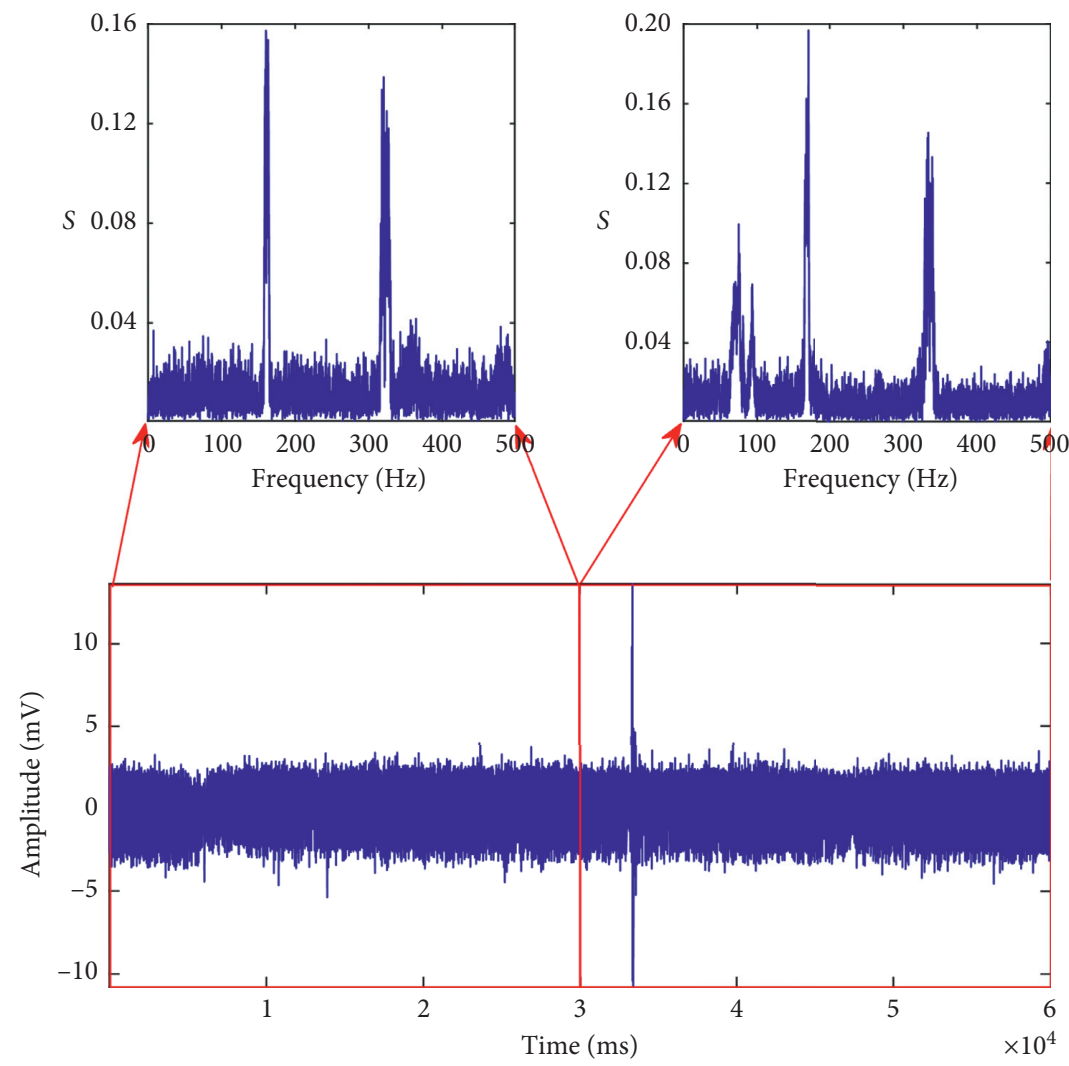

(a)

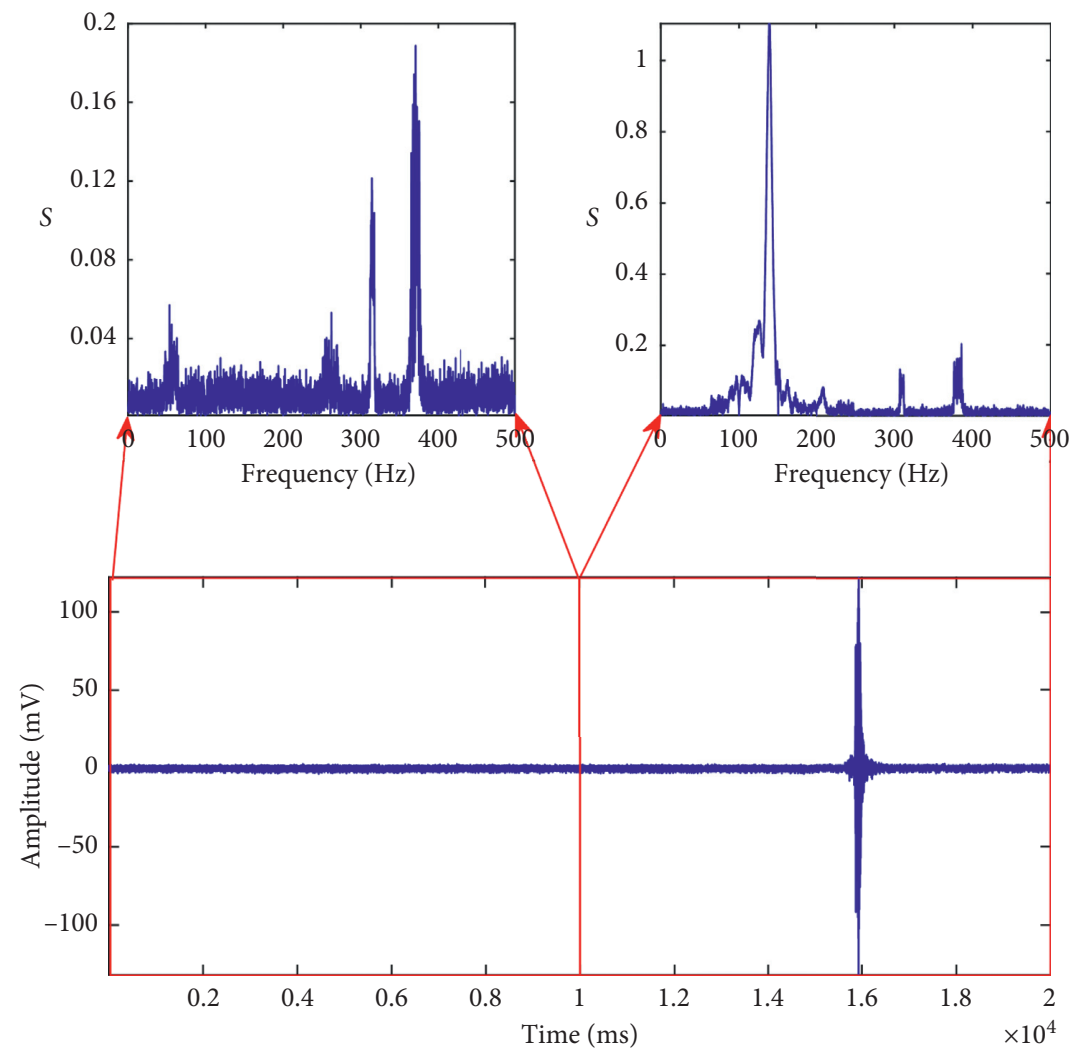

(b)

Figure 1: CSHF signal with (a) low signal-to-noise ratio and (b) high signal-to-noise ratio. 
capability of capturing detailed information with fine resolution in both low-frequency and high-frequency bands. The wavelet packet transform splits the frequency space of a signal into roughly a low-frequency and a high-frequency component using a pair of low pass and high pass filters and then repeats the same splitting procedure for all the obtained components until a specified level. Through this process, the whole frequency space of a signal can be divided into various narrow bands and hence allows better time-frequency localization and frequency components containing low energy to be readily identified [24].

Assume that the initial signal $g_{j}^{n}(t) \in U_{J}^{n}$, and it can be given by $[22,23]$

$$
g_{j}^{n}(t)=\sum_{k} d_{k}^{j, n} u_{n}\left(2^{j} t-k\right)
$$

Thus, the decomposition algorithm for the next subspace $U_{j+1}^{2 n}$ and $U_{j+1}^{2 n+1}$ can be expressed as

$$
\left\{\begin{array}{l}
d_{l}^{j+1,2 n}=\sum_{k} h_{k-2 l} d_{k}^{j, n}, \\
d_{l}^{j+1, n+1}=\sum_{k} g_{k-2 l} d_{k}^{j, n} .
\end{array}\right.
$$

The reconstruction algorithm is

$$
d_{k}^{j, n}=\sum_{l}\left[h_{k-2 l} d_{l}^{j+1,2 n}+g_{k-2 l} d_{l}^{j+1,2 n+1}\right]
$$

where $d_{k}^{j, n}$ are the wavelet coefficients at the level $j, d_{l}^{j+1,2 n}$ and $d_{l}^{j+1,2 n+1}$ are the wavelet coefficients at the next level $j+1$ after decomposition, and $g_{k-2 l}$ and $h_{k-2 l}$ are a pair of low and high pass filters.

After obtaining the wavelet coefficients at each decomposition level, a threshold and thresholding function need to be applied to the coefficients in order to identify and eliminate the frequency components corresponding to noises. Since threshold has a crucial influence on the quality of the reconstructed signal, it is advisable to select an appropriate thresholding criterion for the signals of interest. There are four commonly used threshold criteria for wavelet packet transform: adaptive thresholding (Rigrsure), heuristic thresholding (Heursure), minimaxi thresholding (Minimaxi), and fixed-form thresholding (Sqtwolog) $[25,26]$. The four criteria are based on different rules and thus have their own applicability in signal denoising. Rigrsure features adaptability and uses the soft threshold estimator based on Stein's unbiased risk estimate; Sqtwolog uses fixed-form threshold yielding minimax performance multiplied by a small factor proportional to the signal length; Heursure is a mixture of the two previous criteria; Minimaxi uses a fixed threshold chosen to yield minimax performance for mean square error against an ideal procedure. Minimaxi and Rigrsure tend to be conservative by setting a part of coefficients to zero, and this cannot effectively eliminate all the noise components in the signal of interest, while all the useful information is retained. Heursure and Sqtwolog demonstrate a better capability of identifying the noises components, but in some cases, useful high-frequency components can be mistakenly removed. Therefore, a single global thresholding criterion may not be able to yield desirable denoising results. To illustrate this,
Rigrsure and Sqtwolog are applied and compared to the original signal as shown in Figure 2.

Figure 2 shows that Rigrsure successfully suppresses the background noise at the frequency of $166 \mathrm{~Hz}$, while it fails to remove the noise component at $330 \mathrm{~Hz}$; although Sqtwolog can identify both noise components, it causes distortion between the frequency range of $0-200 \mathrm{~Hz}$. A single global thresholding criterion applied to complex signals, e.g., CSHF signals, may inevitably yield signal distortion or eliminate valid signal components. To enhance the denoising performance for CSHF signals, a multithreshold wavelet packet transform (MWPT) is therefore proposed in this study as a preliminary denoising method. The principles of MWPT are described as follows:

(1) Apply Fourier transform to a CSHF signal $S$, and identify the valid signal's dominant frequency distribution and the noise's frequency distribution.

(2) Determine the optimal wavelet base function and decomposition scale, and implement wavelet packet decomposition with a series of wavelet coefficients obtained.

(3) Apply different thresholding criteria to the wavelet coefficients of different frequency bands: employ the conservative criterion Rigrsure for the valid signal's dominant frequency band to ensure that useful information is retained, e.g., $30-200 \mathrm{~Hz}$ for CSHF signals; the frequency range where noise component is overlapped with partial valid signal component is dealt with using Heursure, e.g., $0-30 \mathrm{~Hz}$ for CSHF signals; apply Sqtwolog to the noise frequency range, e.g., greater than $200 \mathrm{~Hz}$ for CSHF signals.

(4) The denoised wavelet components are then input into the IHHT for twice denoising.

3.2. Improved Hilbert-Huang Transform. Although MWPT allows adaptive thresholding criterion for different frequency bands, it is still incapable of appropriately dealing with those signals whose noise and valid components are partially overlapped without explicit boundaries in the frequency domain. For such signals, using MWPT solely may yield undesirable signal-to-noise ratio or even signal distortion. Therefore, it is recommended to use an improved Hilbert-Huang transform (IHHT) for a twice denoising based on the preliminary denoising results. Hilbert-Huang transform (HHT) has been widely used in analyzing nonlinear and nonstationary signals for its strong adaptability and does not require a priori function basis in the time and frequency domain.

The HHT uses the empirical mode decomposition (EMD) method to adaptively decompose a signal into finite so-called intrinsic mode function (IMF) components that can form a complete and nearly orthogonal basis for the original signal. The obtained IMF components are then used for computing their instantaneous frequency by means of Hilbert transform. The EMD process can be represented as

$$
x(t)=\sum_{i=1}^{n} c_{i}(t)+r_{n}
$$



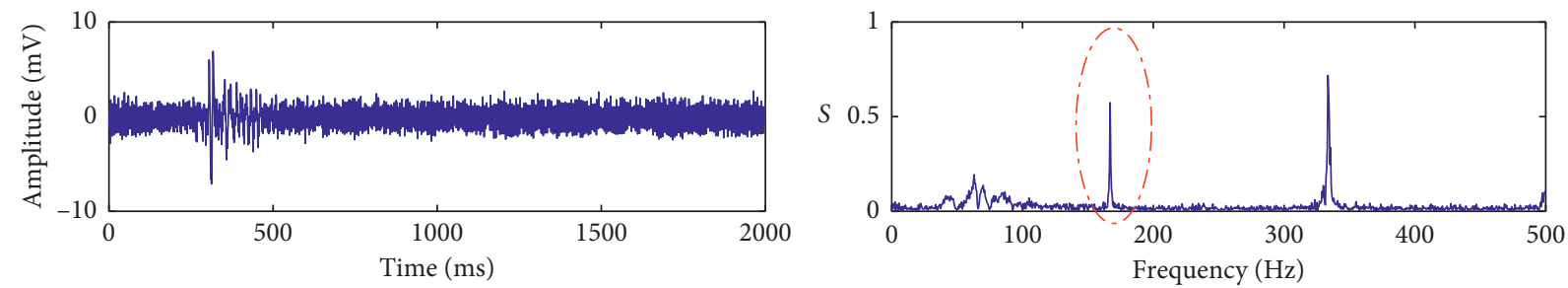

(a)
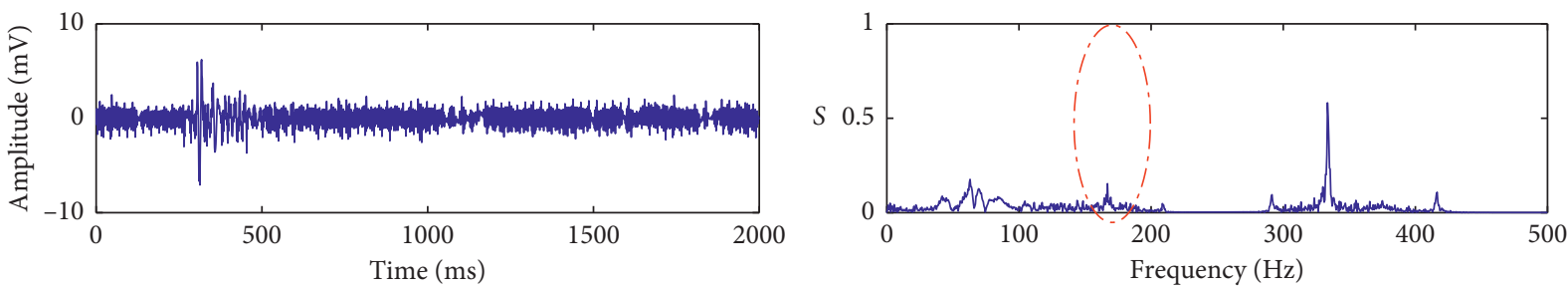

(b)
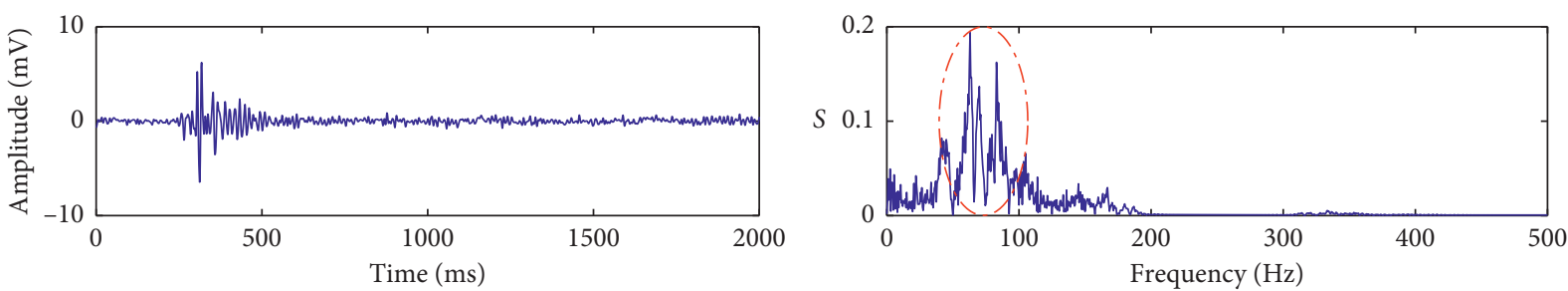

(c)

FIgURe 2: Denoising results in the time-frequency domain: (a) original signal, (b) Rigrsure and (c) Sqtwolog

where $x(t)$ is the input signal, $c_{i}(t)$ is the $i$ th IMF component, and $r_{n}$ is the final residue.

In the conventional HHT method, the stoppage criterion of the sifting process (extracting IMF) is based on a single prescribed threshold of energy ratio or a sum of the difference of IMFs. This type of criterion that selects the first few IMF components in sequence and simply removes the remaining IMFs and the residue may mistakenly "dump" the valid components and retain those redundant IMF components arising from external noises and interference signals. To effectively identify false and redundant IMF components, an improved Hilbert-Huang transform (IHHT) method is therefore introduced by combining the waveform correlation coefficient Corr and the variance contribution rate VCR as the IMF selection criterion.

The waveform correlation coefficient Corr is used as a measure of the correlation between the input signal (denoised wavelet packet coefficient) and its decomposed IMF components, and it can be expressed as

$$
\operatorname{Corr}(i, j)=\frac{\sum_{k=1}^{N}\left[x_{i}(k) c_{(i, j)}(k)\right]}{\sqrt{\sum_{k=1}^{N}\left[x_{i}(k)\right]^{2} \sum_{k=1}^{N}\left[c_{(i, j)}(k)\right]^{2}}}
$$

where $x_{i}(k)$ is the $k$ th sampling point of the $i$ th input wavelet coefficient, $c_{(i, j)}(k)$ is $k$ th sampling point of the $j$ th IMF component obtained from the $i$ th input wavelet packet coefficient, and $N$ is the length of the input signal.
The variance contribution rate VCR reflects the contribution of an IMF component to the input signal, and it can be expressed as

$$
\operatorname{VCR}(i, j)=\frac{\left((1 / N) \sum_{k=1}^{N}\left[c_{(i, j)}(k)\right]^{2}\right)-\left[(1 / N) \sum_{k=1}^{N} c_{(i, j)}(k)\right]^{2}}{\sum_{j=1}^{M}\left\{\left((1 / N) \sum_{k=1}^{N}\left[c_{(i, j)}(k)\right]^{2}\right)-\left[(1 / N) \sum_{k=1}^{N} c_{(i, j)}(k)\right]^{2}\right\}},
$$

where $M$ is the total number of IMF components obtained from the input signal.

The thresholding criterion can be defined as follows: an IMF component is considered as valid when the average of its correlation coefficient and variance contribution rate is greater than a prescribed threshold value. It should be noted that the determination of an appropriate threshold value should be specific to different signal types, i.e., their respective distribution pattern of the correlation coefficient and variance contribution rate for IMF components. For example, for some signals, the Corr and VCR of the first IMF are significantly higher than those of the remaining IMFs, and therefore, a relatively high threshold value should be chosen; if the Corr and VCR concentrate in the first several IMFs, which are moderately higher than those of the remaining IMFs, a moderate threshold value is more appropriate to identify valid IMFs. For the synthetic signals in this study, based on the analysis of the IMFs for all the input wavelet components, 0.4 is selected as the threshold value, whereas 0.95 is selected for the real CSHF signals [6]. 


$$
\frac{\operatorname{Corr}(i, j)+\operatorname{VCR}(i, j)}{2} \geq \lambda_{0}
$$

3.3. Procedure of MWPT-IHHTDenoising Method. A specific program is developed using MATLAB codes to implement the MWPT-IHHT method, and its procedure is summarized in a flow chart (Figure 3 ).

The procedure of the MWPT-IHHT method mainly involves the following steps: (1) wavelet packet decomposition of the input signals; (2) application of the multithreshold criterion to the obtained wavelet coefficients based on the frequency features of the input signal; (3) input of the preliminary denoised wavelet coefficients into the IHHT framework; (4) empirical mode decomposition (EMD) of the input wavelet coefficients and obtaining a series of IMF components; (5) identifying valid IMF components using Corr and VCR; (6) repeating steps (3)-(5) for all the obtained wavelet coefficients; (7) signal reconstruction using all the valid IMF components.

\section{Denoising Performance Evaluation of MWPT-IHHT Using Synthetic Signals}

In order to demonstrate the detailed procedure of the proposed MWPT-IHHT methodg as well as its denoising performance, synthetic signals that are generated based on the Ricker wavelet and the white Gaussian noise are processed by means of the MWPT-IHHT method and then by three commonly used denoising methods for comparison, namely, Hilbert-Huang transform (HHT), wavelet transform (WT), and wavelet packet transform (WPT).

4.1. Generation of Synthetic Signals. The Ricker wavelet that is often used in seismic analysis is introduced here to generate synthetic signals in conjunction with white Gaussian noise as an approximate representation of real CSHF signals. The Ricker wavelet is defined in the time domain as

$$
f(t)=\left(1-2 \pi^{2} f_{p}^{2} t^{2}\right) \exp \left(-\pi^{2} f_{p}^{2} t^{2}\right),
$$

where $f(t)$ is the amplitude, $t$ is the time, and $f_{p}$ is the peak frequency. Here, we choose $f_{p}=35 \mathrm{Hzfor}$ the Ricker wavelets to generate synthetic signals together with the white Gaussian noise, as shown in Figure 4. It is shown that the basic Ricker wavelet has a symmetric trough-crest-trough shape in the time domain with a frequency range of $0-75 \mathrm{~Hz}$. The synthetic signals are then continuously monitored and collected with a sampling frequency of $1000 \mathrm{~Hz}$ for subsequent denoising processing.

4.2. Denoising Procedure of the MWPT-IHHT. To demonstrate how the proposed MWPT-IHHT can be applied in practice, the collected synthetic signals are used for denoising as an example first, and the real CSHF signals captured in field are then examined. The microseismic monitoring system employed in this study has a sampling frequency of $1000 \mathrm{~Hz}$ with a geophone frequency range of $0-500 \mathrm{~Hz}$. Considering that the valid CSHF signals are mainly distributed in the frequency range of $30-200 \mathrm{~Hz}$, the original signals can therefore be decomposed into 5 levels in this case through wavelet packet decomposition technique, and 32 narrow frequency bands can be obtained accordingly denoted by $S_{i}(i=0,1,2, \ldots, 31)$ with each breadth of $15.625 \mathrm{~Hz}$.

To evaluate the noise reduction performance of the MWPT, the first eight frequency bands $S_{0}-S_{7}(0-125 \mathrm{~Hz})$ are selected for denoising, and their frequency spectra before and after denoising are analyzed and illustrated in Figure 5. Blue and red lines represent the original and the denoised frequency bands, respectively. Since the dominant frequency of the Ricker wavelet signals is $35 \mathrm{~Hz}$, the first four frequency bands $S_{0}-S_{4}(0-78.125 \mathrm{~Hz})$ can be considered as the possible frequency range of the valid signals and should be denoised using conservative Rigrsure criterion, while the remaining frequency bands $S_{5}-S_{7}(78.125-125 \mathrm{~Hz})$ mainly comprise noises using Sqtwolog criterion. Figures 5(a)-5(f) show that the extremely small amount of noise in the $S_{0}-S_{4}$ is effectively eliminated on a fine scale after the application of the thresholding criterion of Rigrsure, and relatively smooth frequency spectrum curves are obtained accordingly. Figures 5(g)-5(i) show that all the frequency components in the $S_{5}-S_{7}$ are practically removed by means of Sqtwolog criterion, indicating that the frequency bands for the noise components are successfully filtered. Hence, the use of multiple thresholding criteria for different decomposed frequency bands can considerably enhance complex signals, whereas a global thresholding criterion may not be able to simultaneously suppress noise components in all the frequency bands. It should also be noted that, in Figures 5(c) and 5(d), the presence of local frequency spectrum peaks at the frequency of around $80 \mathrm{~Hz}$ does not coincide with the nature of the Ricker wavelet that should be of a slightly skewed bell shape in the frequency domain. This is probably caused by the nonadaptive splitting of the frequency space during the wavelet packet decomposition, and therefore a twice denoising using the IHHT is critically important.

After applying the MWPT to the input synthetic signal, the obtained frequency bands continue to be processed for a twice denoising using the IHHT. Here, we use the second frequency band $S_{2}(31.25-46.875 \mathrm{~Hz})$ as an example to demonstrate how the IHHT can be applied to further suppress noise in the input frequency bands that have already been preliminarily denoised. The $S_{2}$ is first sequentially decomposed into 6 IMF components and a residue component RES by means of EMD, as illustrated in Figures 6(a)6(g). For IMF1-IMF4 in Figures 6(a)-6(d), the Ricker wavelets that are approximately represented by a symmetric trough-crest-trough shape in the time domain can be distinctly identified, and their frequencies mainly concentrate in the range of $0-50 \mathrm{~Hz}$, which is consistent with that of the designed Ricker wavelet. However, the Ricker wavelets can hardly be identified for the remaining IMF components with their dominant frequency being approaching zero in the frequency spectrum. Thus, IMF1-IMF4 can be intuitively considered as valid. 


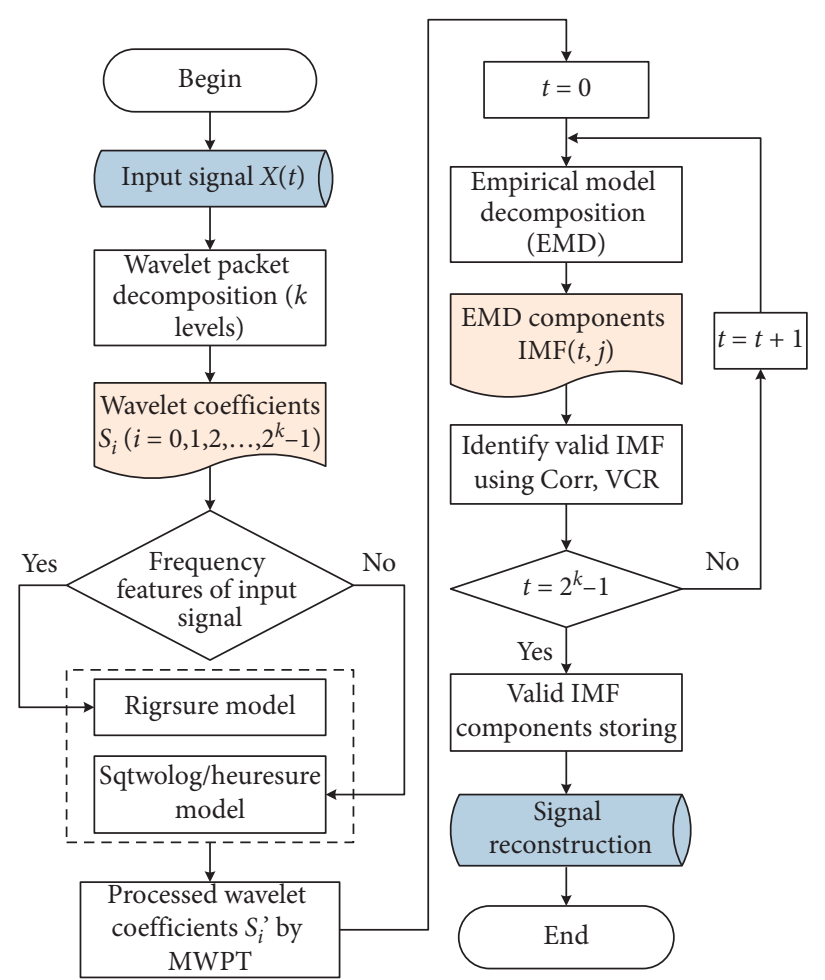

Figure 3: Flow chart of the MWPT-IHHT.
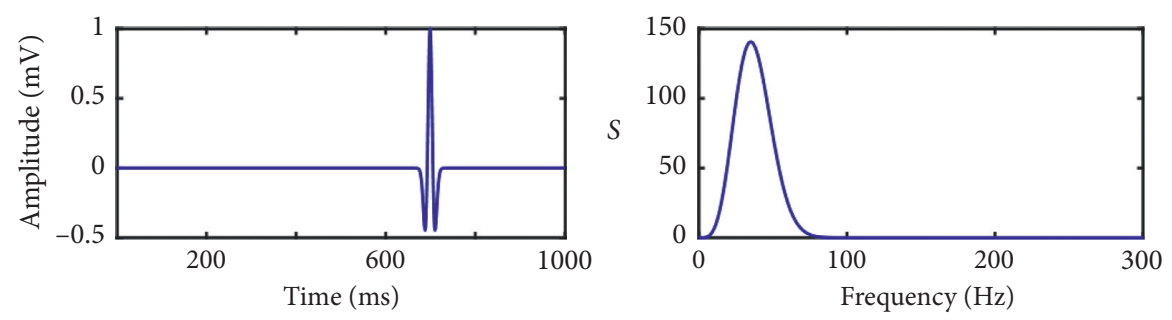

(a)
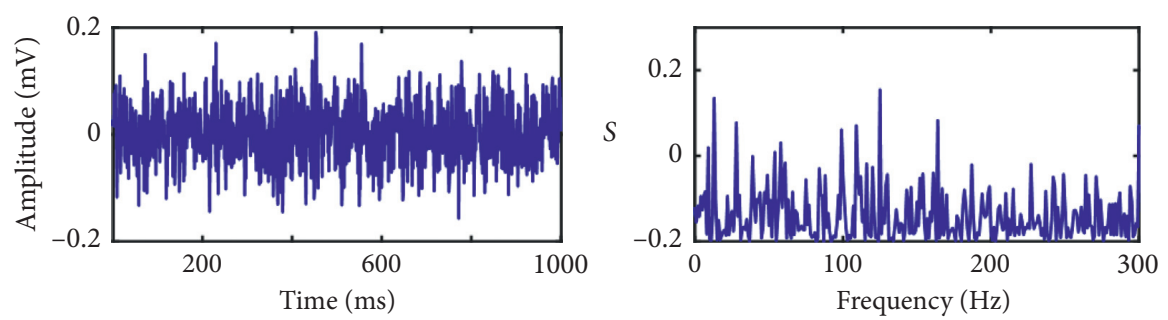

(b)
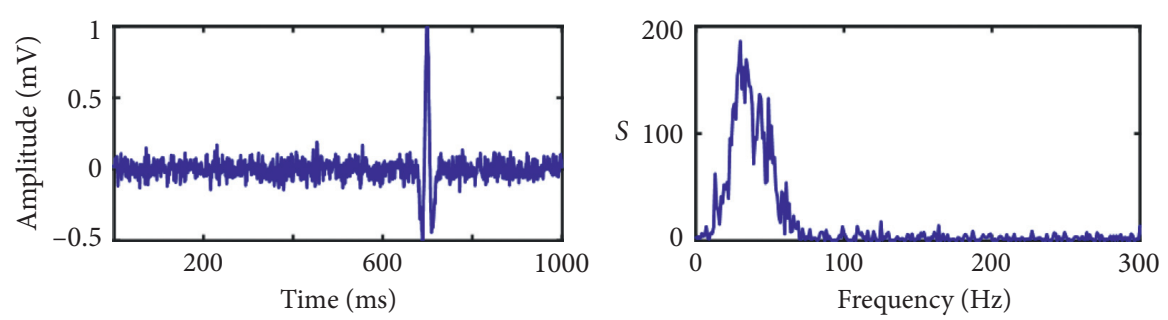

(c)

FIgURE 4: Synthetic signals: (a) Ricker wavelet, (b) white Gaussian noise, and (c) synthetic signal. 


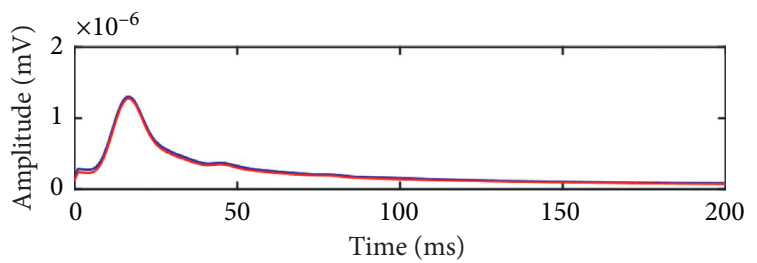

(a)

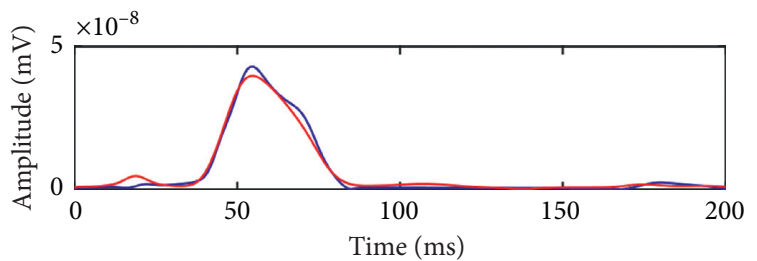

(c)

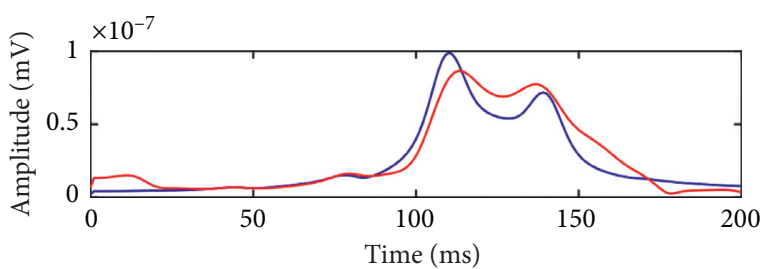

(e)

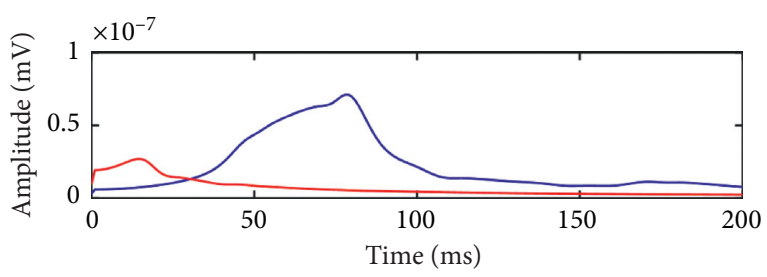

(g)

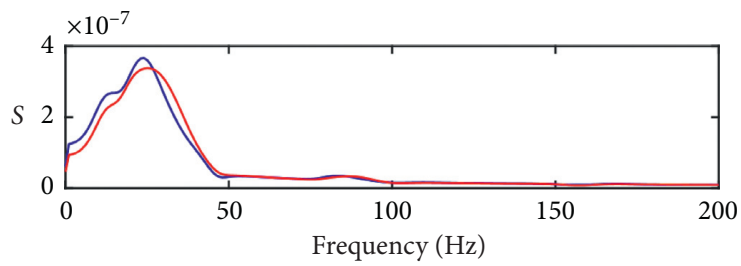

(b)

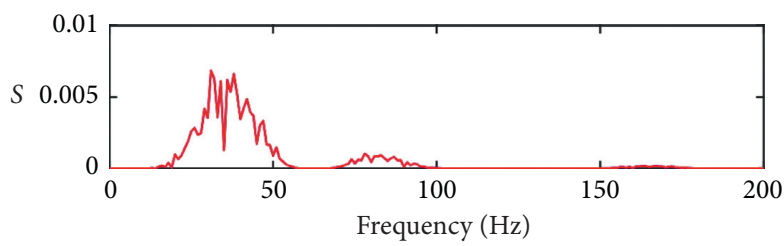

(d)

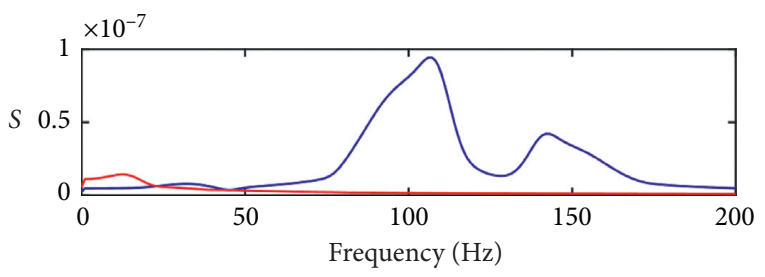

(f)

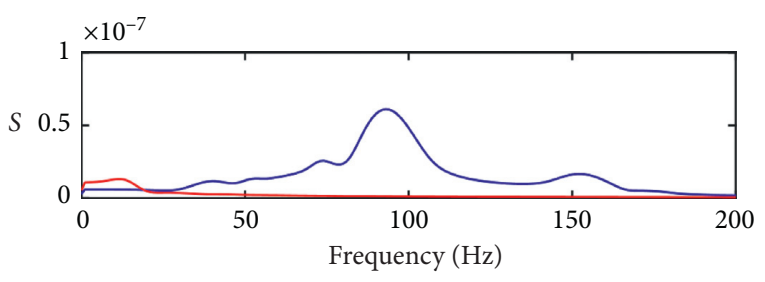

(h)

FIGURE 5: Illustration of MWPT denoising performance for $S_{1}-S_{7}$ frequency bands.
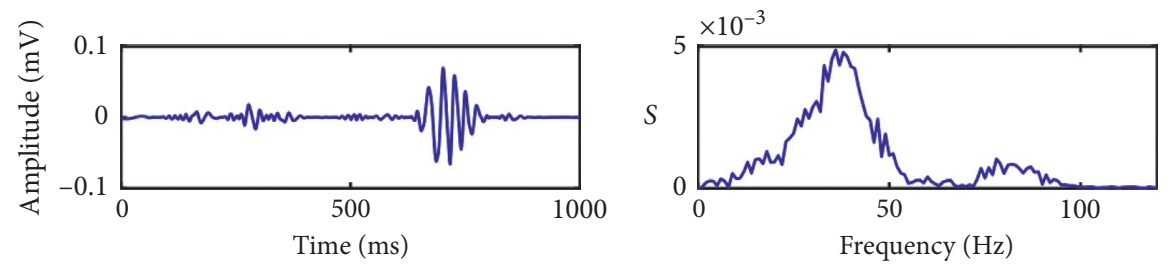

(a)
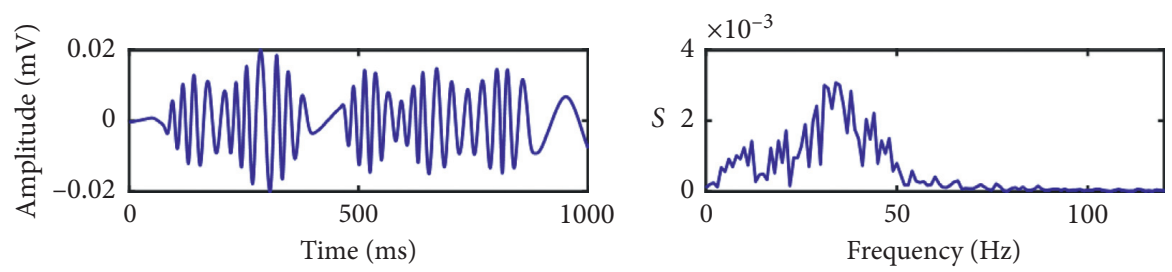

(b)

Figure 6: Continued. 

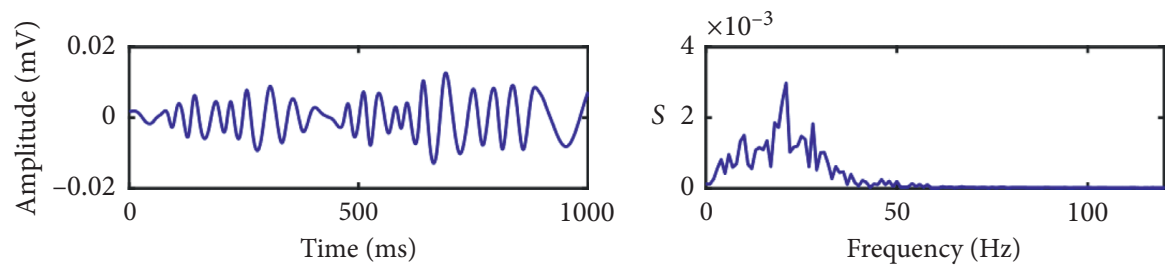

(c)
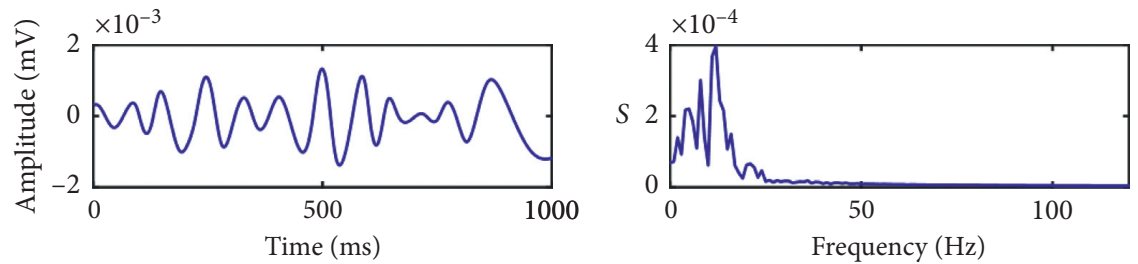

(d)
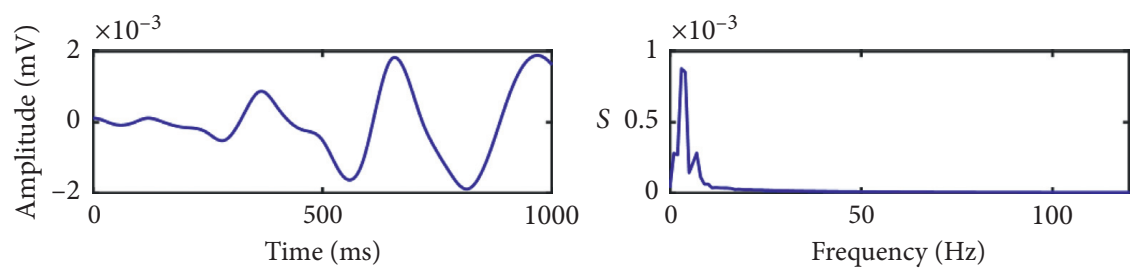

(e)
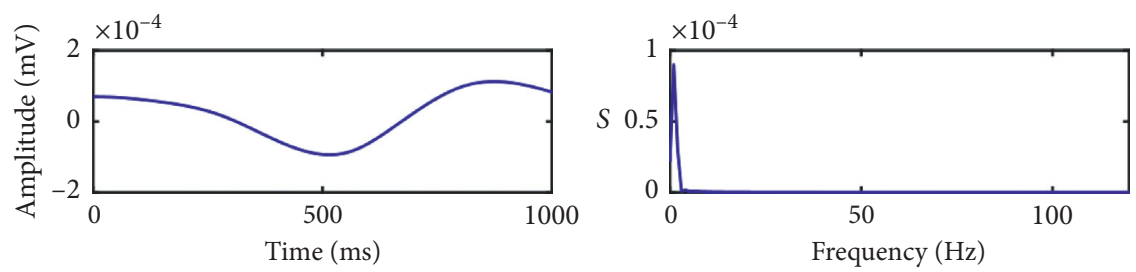

(f)
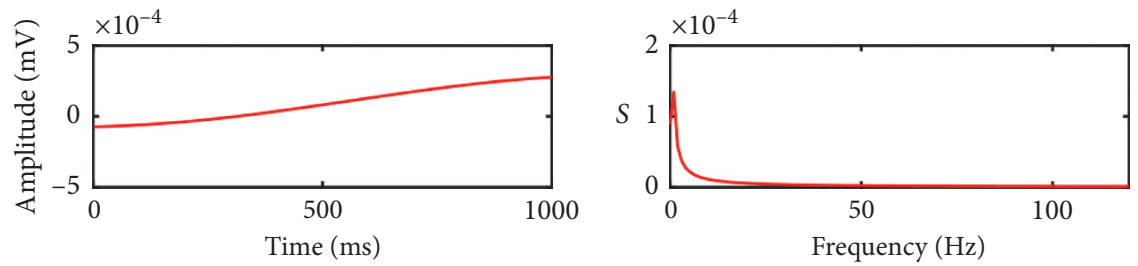

(g)

FIGURE 6: IMF components of the $S_{2}$ frequency band.

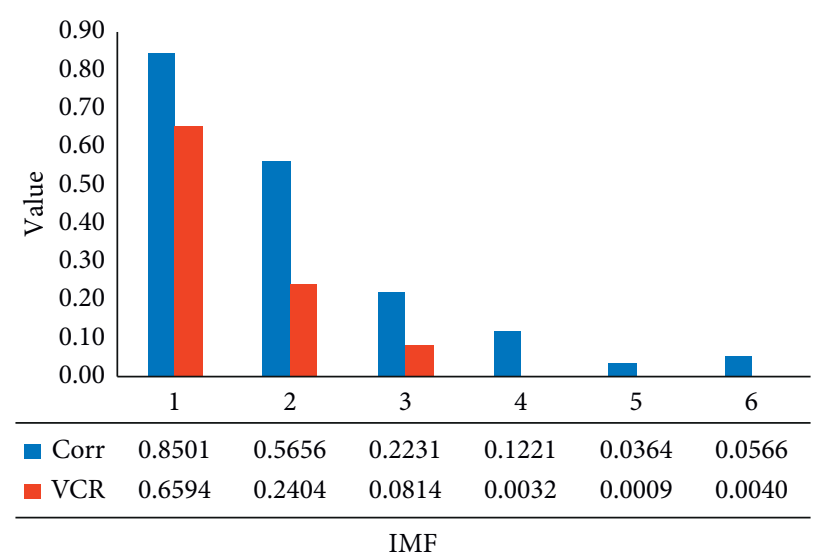

FIGURE 7: Correlation coefficient and variance contribution rate for IMF components. 
To eliminate the redundant IMF components in a quantitative manner, the waveform correlation coefficient Corr, the variance contribution rate VCR, and their average are calculated using eqs. (5)-(7), respectively. Figure 7 shows that the first two IMFs have substantially higher Corr and VCR than those of other IMFs with their respective average greater than 0.4 and are thus considered as valid and selected for subsequent signal reconstruction.

4.3. Denoising Performance Evaluation of MWPT-IHHT. To evaluate the denoising performance, the proposed MWPT-IHHT method is compared with the three commonly used denoising methods-namely, HHT, WT, and WPT-in the 3- dimensional time-frequency domain.

Figure 8 shows the denoising results of a synthetic signal in the time-frequency domain using the three commonly used denoising methods and the proposed MWPT-IHHT method. The original signal in Figure 8(a) has a rather low signal-to-noise ratio in the sense that a large amount of noise spreads over the frequency band of approximately $200-500 \mathrm{~Hz}$. Intuitively, the HHT has the poorest performance in suppressing the noise components, whereas both the WT and WPT yield better denoising results, with fewer noise components being observed overall. However, for the WT, a frequency concentration out of the dominant frequency range is observed in the high-frequency band $(300-500 \mathrm{~Hz})$ that is inconsistent with the original signal. Additionally, the poor resolution in the dominant frequency range has artificially led to an increase in the area of the valid signals in the time-frequency domain, which may cause an undesirable identification of nearby noise components. Due to the lack of adaptivity of the WPT, the noise components in the overlapping frequency range with the valid signal cannot be effectively eliminated. The proposed MWPT-IHHT method, from the display in the time-frequency domain, appears to have the capability of overcoming these shortcomings in terms of both retaining the valid signal in a fine manner and effectively suppressing noise components.

To further demonstrate MWPT-IHHT's outperformance over the three methods in signal denoising, the signal-to-noise ratio SNR, the root-mean-square error RMSE, and the energy ratio ER are employed for quantitative comparison and evaluation. RMSE is a measure of the similarity between the denoised signal and the original signal, and ER is a measure of the contribution in the denoised signal from the original signal. It should be noted that higher SNR, ER, and lower RMSE are interpreted as more desirable denoising performance. The three indices of interest are given by

$$
\begin{aligned}
\mathrm{SNR} & =10 \lg \left\{\frac{\sum_{n=1}^{N} \widehat{S}_{n}^{2}}{\sum_{n=1}^{N}\left[\left(S_{n}-\widehat{S}_{n}\right)^{2}\right]}\right\}, \\
\mathrm{RMSE} & =\frac{\sqrt{\sum_{n=1}^{N}\left[\left(S_{n}-\widehat{S}_{n}\right)^{2}\right]}}{N}
\end{aligned}
$$

$$
\mathrm{ER}=\frac{\sum_{n=1}^{N}\left(\widehat{S}_{n}\right)^{2}}{\sum_{n=1}^{N}\left(S_{n}\right)^{2}}
$$

where $N$ is the number of sampling points; $S_{n}$ and $\widehat{S}_{n}$ represent the signal before and after denoising, respectively.

Table 1 shows the three indices for each denoising method of interest. It is seen that both the SNR and ER for HHT are significantly lower than those for other methods, indicating a relatively poor denoising result that is consistent with the intuitive interpretation from the 3-dimensional display in the time-frequency domain. The WT exhibits its advantages over the WPT in terms of the SNR, whereas the WPT has a moderately better performance in terms of both RMSE and ER. However, neither of them yields desirable results for all the three indices. It can be readily concluded that the proposed MWPT-IHHT has the most desirable denoising performance that yields the highest SNR and lowest RMSE with a relatively higher ER. It should be noted that the SNR, an essential index for evaluating denoising performance, has been significantly enhanced by approximately 3 times using the MWPT-IHHT method compared to the HHT method.

\section{Engineering Application of MWPT-IHHT for CSHF Signals}

The purpose of mine microseismic signal (MMS) denoising is to extract the effective signal from background interference and can be further used in wave classification and identification, time pickup, location calculation, mining feature detailing, etc. To demonstrate the applicability of the proposed MWPT-IHHT method for CSHF signals in practice, the microseismic signals captured in the CSHF test at Huafeng Coal Mine in China are used for verification. This CSHF test was conducted in a longwall panel at a depth of around $1000 \mathrm{~m}$ with the purpose of regional rockburst prevention.

5.1. Denoising Result of CSHF Signals. For brevity, two typical CSHF signals with high and low SNR are selected for demonstrating the applicability and effectiveness of the proposed MWPT-IHHT denoising method, as illustrated in Figure 9. The left and right diagrams represent the original and denoised signal in the time and frequency domain, respectively. For signal \#1, the noise components are effectively suppressed in the sense that the valid signals become far more distinguishable in the time domain, and the fake frequency components out of the dominant frequency range are greatly reduced. For signal \#2, the weak valid component that is smeared in the relatively strong background noises can still be reasonably identified and extracted, in which two prominent frequency peaks representing noises are appropriately eliminated. This demonstrates the applicability of the proposed MWPT-IHHT method for denoising real CSHF signals and thus can lay the foundation for further high-precision source locating. 


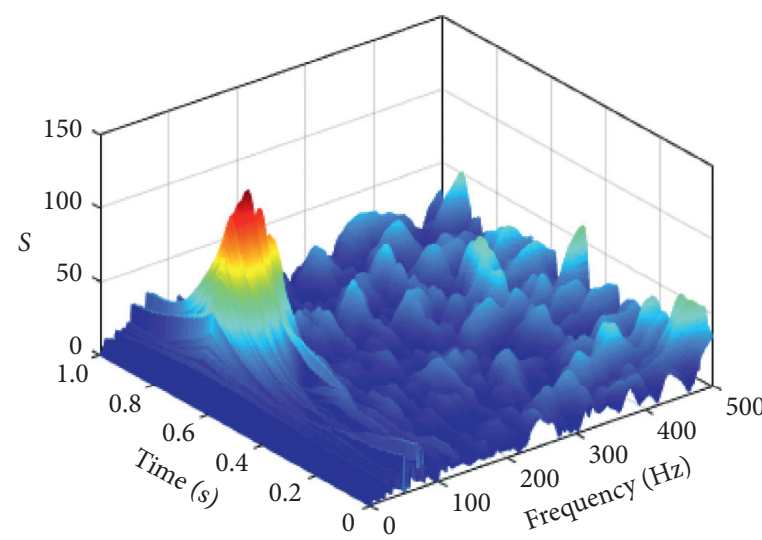

(a)

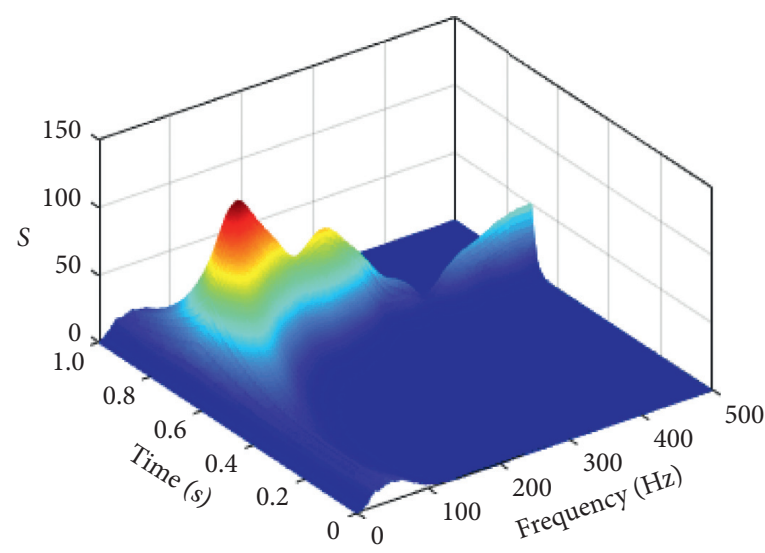

(c)

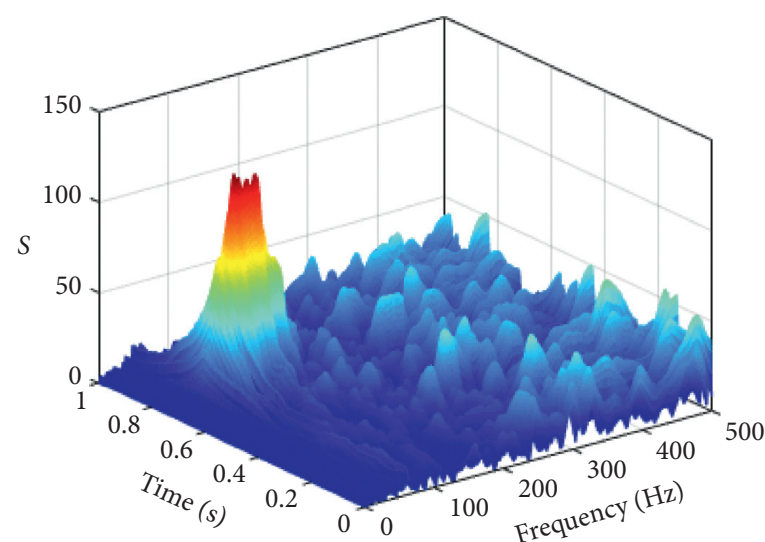

(b)

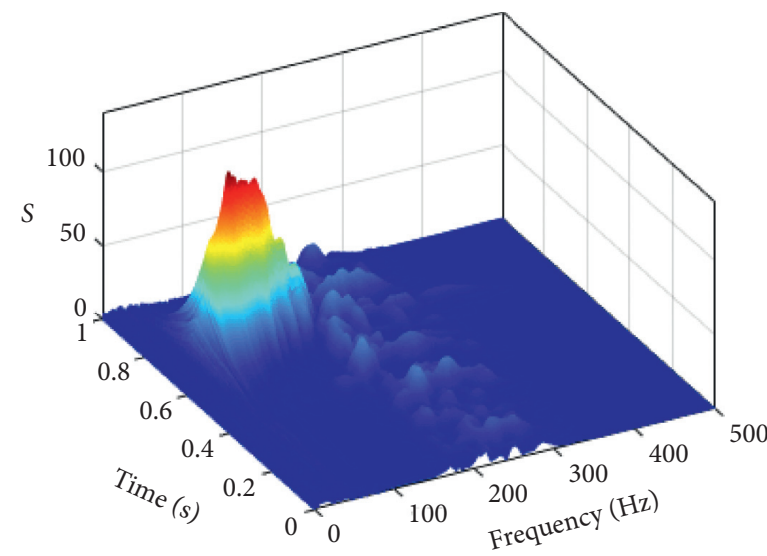

(d)

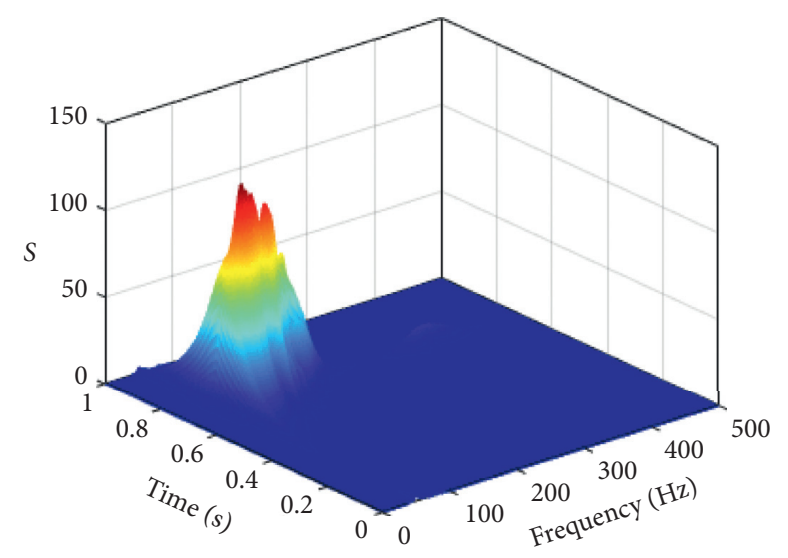

(e)

FIGURE 8: Time-frequency comparison of the MWPT-IHHT and three commonly used denoising methods. (a) Original synthetic signal. (b) HHT. (c) WT. (d) WPT. (e) MWPT-IHHT.

TABLE 1: Quantitative comparison of four denoising methods.

\begin{tabular}{lccc}
\hline Method & SNR & RMSE (\%) & ER (\%) \\
\hline HHT & 12.72 & 1.88 & 79.42 \\
WT & 30.00 & 3.45 & 84.47 \\
WPT & 24.71 & 2.26 & 93.54 \\
MWPT-IHHT & 31.53 & 0.40 & 92.20 \\
\hline
\end{tabular}



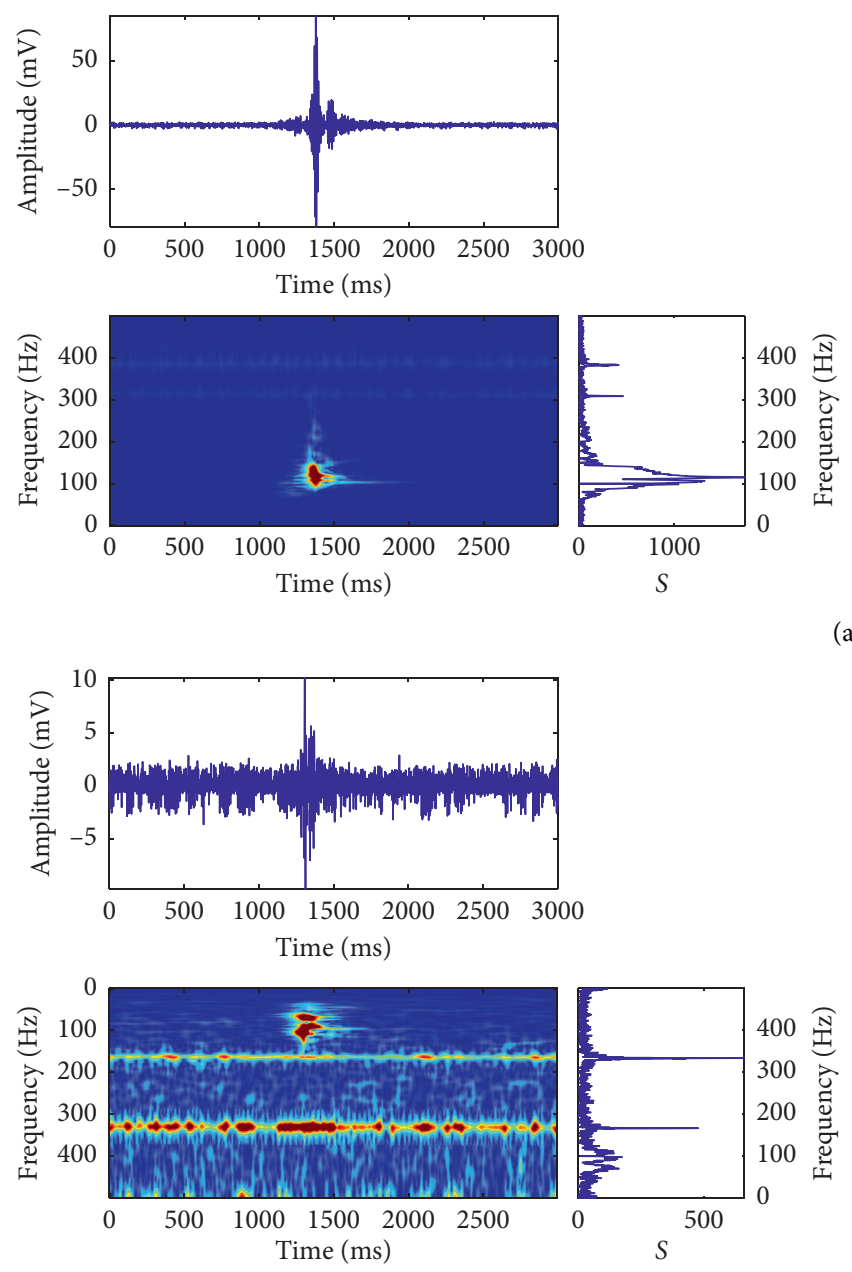

(a)
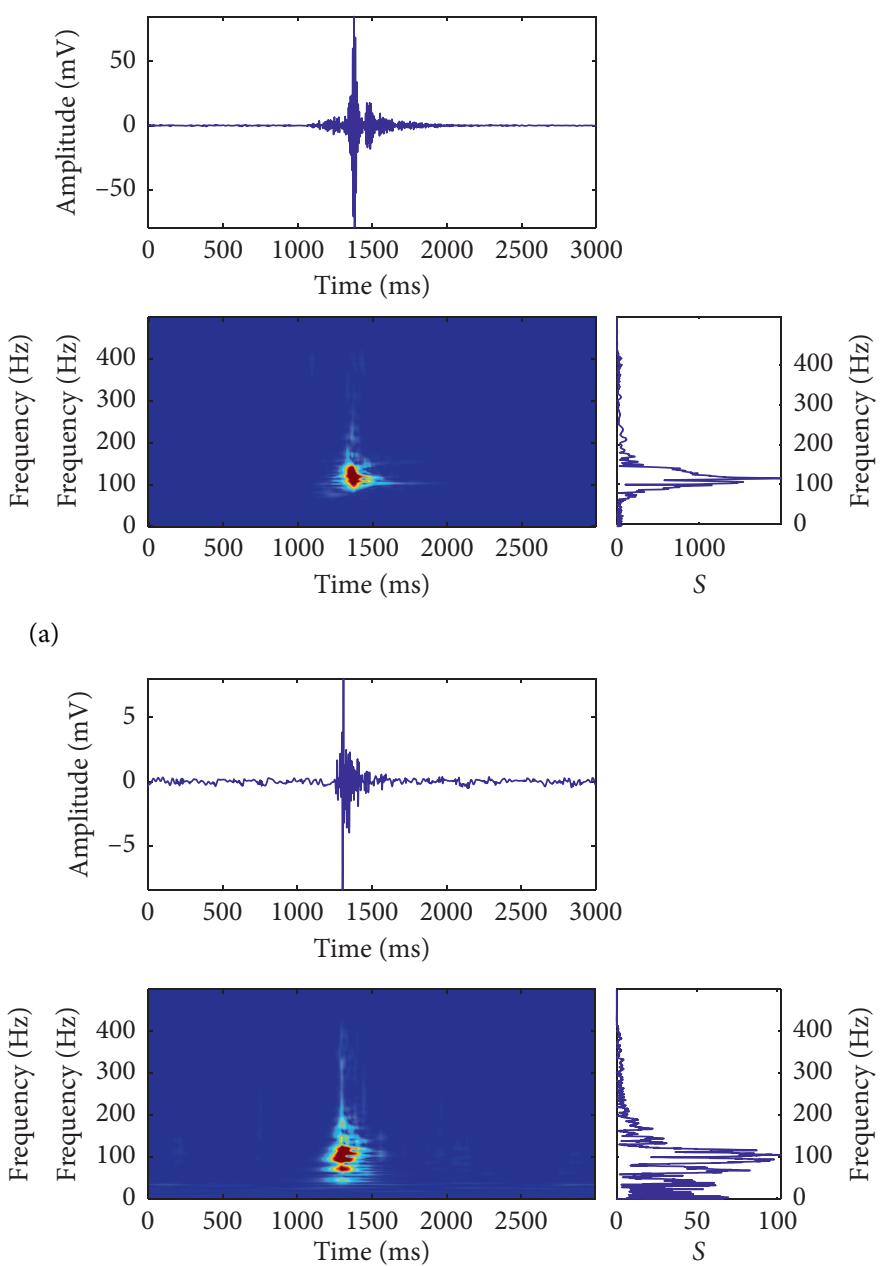

(b)

Figure 9: MWPT-IHHT method applied to real CSHF signals. (a) Signal \#1 with high SNR. (b) Signal \#2 with low SNR.

5.2. MS Mechanisms and Moment Tensors. The MS sources locations visually correlated with crack initiation and extension inside the coal and reflected the variation of coal and rock degradation and failure. MS parameter analysis can be used to evaluate crack initiation and extension based on the MS monitoring waveforms and parameters such as energy (magnitude), count rate, event occurrence, and spatial distribution. But event locations and magnitudes give limited insight into the processes that control the growth and dynamics of hydraulic fractures. The reservoir behavior can be enhanced by considering the seismic moment tensor representations of these events, which serve as a direct snapshot of the instantaneous deformation of the surrounding rock by the seismicity. Therefore, by using moment tensor (MT) analysis, the cracking and damage mechanisms in the coal can be quantitatively estimated during the CSHF process.

After denoising the signals, source locations were initially estimated using first compressional arrival times with a constant, homogeneous, and isotropic velocity model. And an optimized location algorithm was used to improve positioning accuracy (more details of this test can be found in [6]). A typical CSHF signal after preprocessing and associated parameters for MTs calculation are shown in Figure 10.

Based on the data preprocess, moment tensor components (MTs) were calculated [27, 28] and provided quantitative information on MS location of cracks, crack type, crack orientation, and the direction of crack motion. A source-type diagram (Hudson plot) was introduced as a helpful way to display the results of MTs. Hudson et al. [29] introduced a diamond $\tau-k$ plot of source-type, which is the diamond CLVD-ISO plot described in the previous section but with the opposite direction of the CLVD axis and a skewed diamond $u-v$ plot. The latter plot is introduced in order to conserve the uniform probability of moment tensor eigenvalues. The data generated by the CSHF test at Huafeng Coal Mine in China is being studied. The MS monitoring was undertaken for the whole process. During that time period, hundreds of MS events were records. In Figure 11, the result solutions were decomposed following Hudson's method, and results could be observed on Hudson's plot.

As shown above, the occurrence mechanisms of fracturing-induced, natural, and mining-induced microseismic events are different. The failure mechanisms in different stage have high disparities. In stage of crack extension stage 


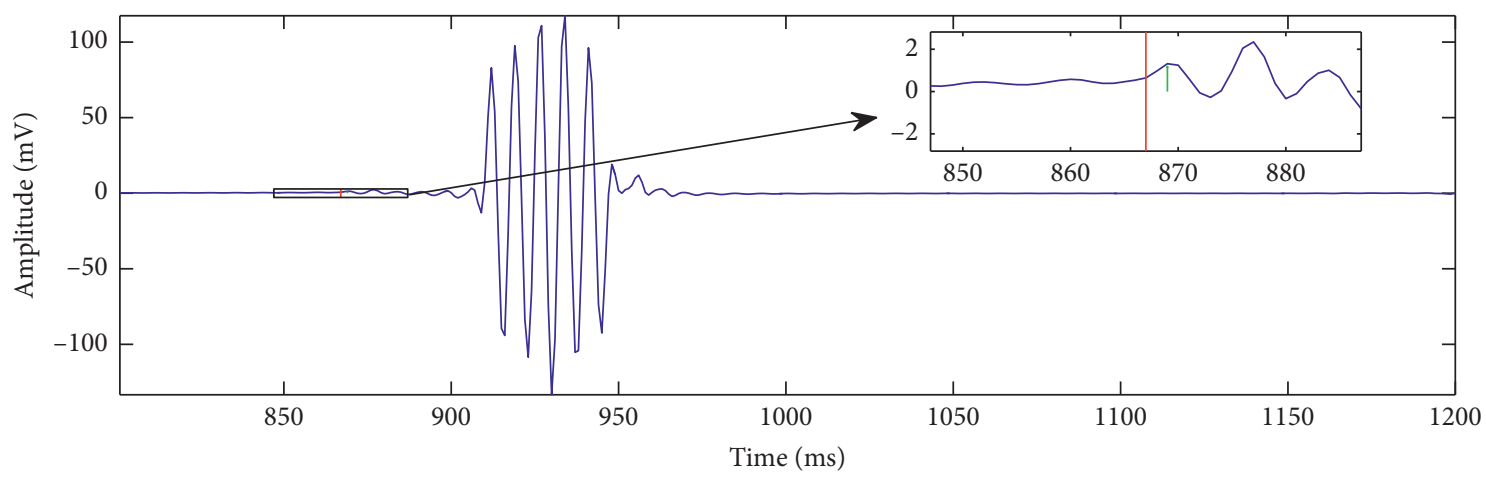

$$
\begin{aligned}
& \longrightarrow \text { DS } \\
& - \text { FAT } \\
& \longrightarrow \text { IMA }
\end{aligned}
$$

FIGURE 10: Typical preprocess signal of CSHF (blue line) and associated parameters for MTs calculation (first arrival time in red line, initial motion amplitude in green line).

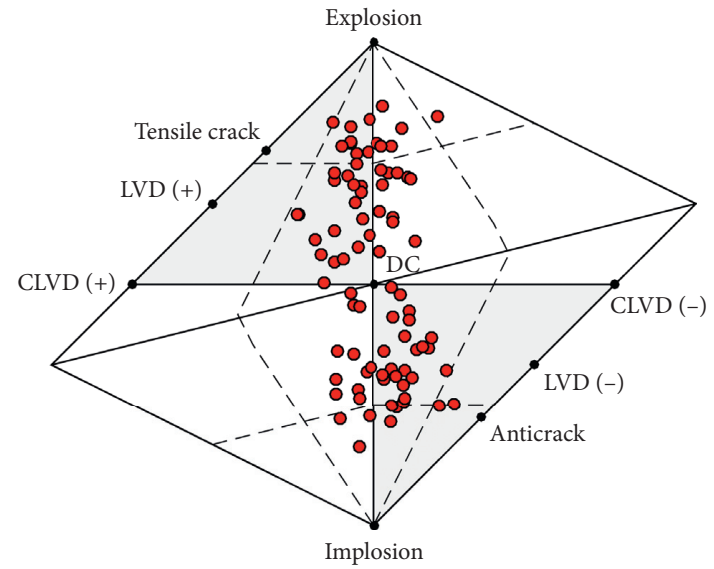

(a)

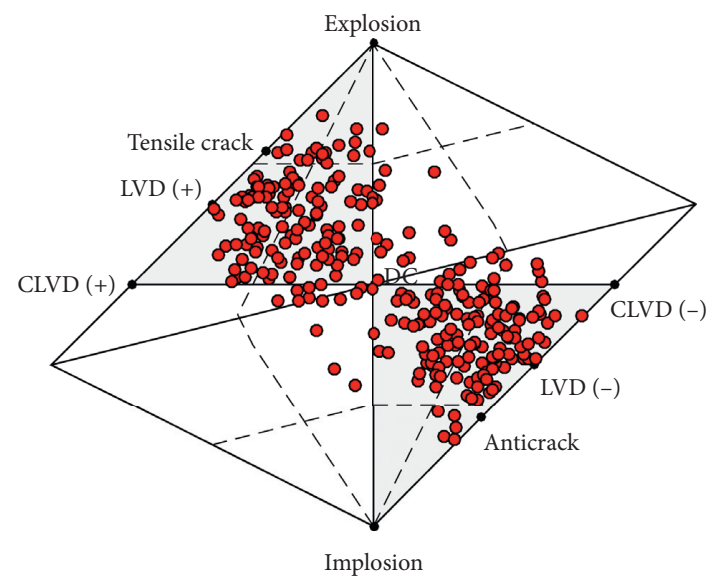

(c)

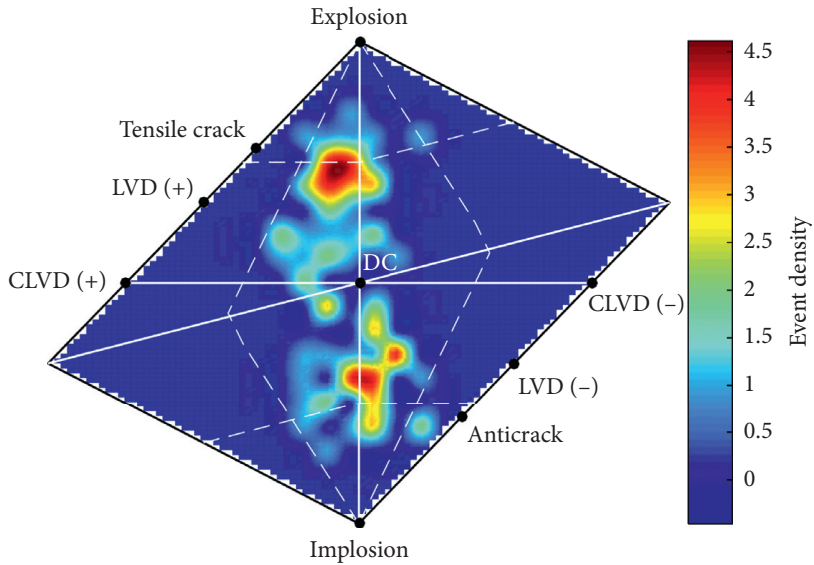

(b)

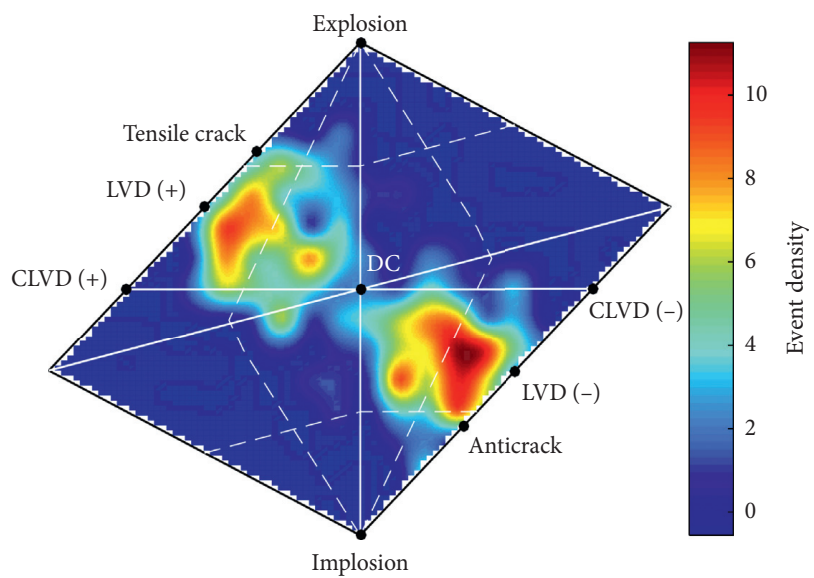

(d)

FIGURE 11: Scatter and density source plots of the MS events with Hudson diagrams (T-k plot). (a, c) Scatter plot of MS events. (b, d) Density source plot of MTs from MS data (colored according to relative seismic event density). (a) Scatter plot (crack extension stage). (b) Density source plots (crack extension stage). (c) Scatter plot (crack open/close stage). (d) Density source plots (crack open/close stage). 
(Figures 11(a) and 11(b)), diamond-shaped plots show MS events around the central axis. The majority of the selected events show positive Explosive and Implosive components, which are correlated with each other. This pattern indicated that the failure mechanisms were primarily explosive and implosive, coupled with pure shearing. In the stage of crack open/close (Figures 11(c) and 11(d)), MS events can be found on the upper-left and lower-right of the plot. Such pattern in the source-type plot agrees with the theoretical modeling of the shear-tensile source mechanisms and indicates the influence of massive fluid injection in this area. In hydraulic fracturing, microseismic events are mainly generated once the effective tensile stress induced by high-pressure water reaches the coal's tensile strength.

It can be seen from the above analysis that the source-type plot can show a major failure mechanism of CSHF. This procedure allowed us to determine the proportion of shear and tensile crack contributions on the MS sources and to classify cracks by types of dominant motion. Thus, it can be used to provide many important information and support applications aimed at providing operators with tools to optimize production. In the next step, we will use this method focusing on description and evaluation quantitatively of CSHF.

\section{Conclusions}

The conventional denoising methods-HHT, WT, and WPT-are not well-suited for CSHF signals due to their transient, nonstationary, low energy, and low SNR characteristics. Therefore, combining the multithreshold WPT method and the improved HHT method, the MWPT-IHHT method for twice denoising is proposed, with each being improved compared to their conventional counterpart. Several conclusions can be drawn from this study as follows:

(1) In the MWPT method, multiple thresholding criteria applied to the wavelet coefficients of different frequency bands can avoid the deficiency of a single global thresholding criterion, which, in some cases, may inevitably eliminate valid signal components.

(2) In the IHHT method, the introduction of the waveform correlation coefficient and the variance contribution rate combined as the IMF selection criterion can effectively overcome the deficiency of the conventional HHT method that may mistakenly "dump" valid IMF components and retain redundant ones.

(3) The combination of the MWPT and IHHT significantly enhances the time-frequency localization and allows CSHF signals to be processed in a fine manner. The MWPT-IHHT method can thus overcome each submethod's deficiency-lack of adaptivity and insufficient resolution in the frequency domain.

(4) The quantitative comparison using synthetic signals suggests the superiority of the MWPT-IHHT method over the commonly used denoising methods (HHT,
WT, and WPT) in suppressing noises in terms of SNR, RMSE, and ER.

(5) The desirable denoising results of two typical real CSHF signals with high and low SNR demonstrate the applicability and effectiveness of the MWPTIHHT method for processing CSHF signals. And the source-type plot shows a major shear-tensile mechanism using denoising signals.

\section{Data Availability}

The data used to support the findings of the study are available from the corresponding author upon request.

\section{Conflicts of Interest}

The authors declare that they have no conflicts of interest.

\section{Acknowledgments}

This work was supported by and financed from National Key Research and Development Program of China (Nos. 2017YFC0804603 and 2018YFC0808402), the National Natural Science Foundation of China (No. 51604115), and the Foundation of Hebei Higher Education Institutions (Z2020124).

\section{References}

[1] G. Bräuner, Rockbursts in Coal Mines and Their Prevention, Routledge, Abingdon, UK, 2017.

[2] P. K. Kaiser and M. Cai, "Design of rock support system under rockburst condition," Journal of Rock Mechanics and Geotechnical Engineering, vol. 4, no. 3, pp. 215-227, 2012.

[3] J.-c. Wang, F.-x. Jiang, X.-j. Meng, X.-y. Wang, S.-t. Zhu, and Y. Feng, "Mechanism of rock burst occurrence in specially thick coal seam with rock parting," Rock Mechanics and Rock Engineering, vol. 49, no. 5, pp. 1953-1965, 2016.

[4] J. Zhang, F. Jiang, S. Zhu, and L. Zhang, "Width design for gobs and isolated coal pillars based on overall burst-instability prevention in coal mines," Journal of Rock Mechanics and Geotechnical Engineering, vol. 8, no. 4, pp. 551-558, 2016.

[5] S. Zhu, Y. Feng, and F. Jiang, "Determination of abutment pressure in coal mines with extremely thick alluvium stratum: a typical kind of rockburst mines in China," Rock Mechanics and Rock Engineering, vol. 49, no. 5, pp. 1943-1952, 2016.

[6] Q. Zhu, Y. Feng, M. Cai, J. Liu, and H. Wang, "Interpretation of the extent of hydraulic fracturing for rockburst prevention using microseismic monitoring data," Journal of Natural Gas Science and Engineering, vol. 38, pp. 107-119, 2017.

[7] J. D. Bredehoeft, R. G. Wolff, W. S. Keys, and E. Shuter, "Hydraulic fracturing to determine the regional in situ stress field, Piceance Basin, Colorado," Geological Society of America Bulletin, vol. 87, no. 2, pp. 250-258, 1976.

[8] M. K. Hubbert and D. G. Willis, "Mechanics of hydraulic fracturing," Transactions of the AIME, vol. 210, no. 01, pp. $153-168,1957$.

[9] C. T. Montgomery and M. B. Smith, "Hydraulic fracturing: history of an enduring technology," Journal of Petroleum Technology, vol. 62, no. 12, pp. 26-40, 2010.

[10] J. Fan, L. Dou, H. He et al., "Directional hydraulic fracturing to control hard-roof rockburst in coal mines," International 
Journal of Mining Science and Technology, vol. 22, no. 2, pp. 177-181, 2012.

[11] H. He, L. Dou, J. Fan, T. Du, and X. Sun, "Deep-hole directional fracturing of thick hard roof for rockburst prevention," Tunnelling and Underground Space Technology, vol. 32, pp. 34-43, 2012.

[12] R. Jeffrey and K. Mills, "Hydraulic fracturing applied to inducing longwall coal mine goaf falls," in Proceedings of the 4th North American Rock Mechanics Symposium, pp. 423-430, American Rock Mechanics Association, Seattle, DC, USA, July 2000.

[13] T. Bartosch and D. Seidl, "Spectrogram analysis of selected tremor signals using short-time Fourier transform and continuous wavelet transform," Annals of Geophysics, vol. 42, no. 3, 1999.

[14] B. Boashash and P. Black, "An efficient real-time implementation of the wigner-ville distribution," IEEE Transactions on Acoustics, Speech, and Signal Processing, vol. 35, no. 11, pp. 1611-1618, 1987.

[15] A. Chakraborty and D. Okaya, "Frequency-time decomposition of seismic data using wavelet-based methods," Geophysics, vol. 60, no. 6, pp. 1906-1916, 1995.

[16] N. E. Huang and Z. Wu, "A review on Hilbert-Huang transform: method and its applications to geophysical studies," Reviews of Geophysics, vol. 46, 2008.

[17] S. Parolai, "Denoising of seismograms using the S transform," Bulletin of the Seismological Society of America, vol. 99, no. 1, pp. 226-234, 2009.

[18] Q. Zhu, F. Jiang, Z. Yu, Y. Yin, and L. Lu, "Study on energy distribution characters about blasting vibration and rock fracture microseismic signal," Chinese Journal of Rock Mechanics and Engineering, vol. 31, pp. 723-730, 2012.

[19] R. Kimiaefar, H. Siahkoohi, A. Hajian, and A. Kalhor, "Seismic random noise attenuation using artificial neural network and wavelet packet analysis," Arab J Geosci, vol. 9, pp. 1-11, 2016.

[20] B. M. Battista, C. Knapp, T. McGee, and V. Goebel, “Application of the empirical mode decomposition and hilberthuang transform to seismic reflection data," Geophysics, vol. 72, no. 2, pp. H29-H37, 2007.

[21] R. R. Zhang, S. Ma, E. Safak, and S. Hartzell, "Hilbert-Huang transform analysis of dynamic and earthquake motion recordings," Journal of Engineering Mechanics, vol. 129, no. 8, pp. 861-875, 2003.

[22] R. R. Coifman, Y. Meyer, S. Quake, and M. V. Wickerhauser, Signal Processing and Compression with Wavelet Packets. in: Wavelets and Their Applications, Springer, Berlin, Germany, 1994.

[23] M. V. Wickerhauser, Acoustic Signal Compression with Wavelet Packets. in: Wavelets: A Tutorial in Theory and Applications, Academic Press Professional, Inc., Cambridge, MA, USA, 1993.

[24] L.-S. Law, J. H. Kim, W. Y. H. Liew, and S.-K. Lee, “An approach based on wavelet packet decomposition and hilberthuang transform (WPD-HHT) for spindle bearings condition monitoring," Mechanical Systems and Signal Processing, vol. 33, pp. 197-211, 2012.

[25] D. L. Donoho, "De-noising by soft-thresholding," IEEE Transactions on Information Theory, vol. 41, no. 3, pp. 613627, 1995.

[26] D. L. Donoho and I. M. Johnstone, "Ideal spatial adaptation by wavelet shrinkage," Biometrika, vol. 81, no. 3, pp. 425-455, 1994.
[27] J. Du, U. Zimmer, and N. Warpinski, "Fault-plane solutions from moment tensor inversion for microseismic events using single-well and multi-well data," CSEG Recorder, vol. 10, pp. 22-29, 2011.

[28] I. V. Rodriguez, S. Stanchits, and J. Burghardt, "Data-driven, in situ, relative sensor calibration based on waveform fitting moment tensor inversion," Rock Mechanics \& Rock Engineering, vol. 50, pp. 1-21, 2017.

[29] J. A. Hudson, R. G. Pearce, and R. M. Rogers, "Source type plot for inversion of the moment tensor," Journal of Geophysical Research, vol. 94, no. B1, pp. 765-774, 1989. 